\title{
Optimal Foraging for Multiple Resources in Several Food Species
}

\author{
Geerten M. Hengeveld, ${ }^{1,2,3,}$ Frank van Langevelde, ${ }^{2}$ Thomas A. Groen, ${ }^{2,4}$ and Henrik J. de Knegt ${ }^{2}$
}

1. Department of Plant-Animal Interactions, Netherlands Institute of Ecology (NIOO-KNAW), Centre for Limnology, Rijksstraatweg 6, 3631 AC Nieuwersluis, The Netherlands; 2. Resource Ecology Group, Wageningen University, Droevendaalsesteeg 3a, 6708 PB Wageningen, The Netherlands; 3. Team Forest Ecology, Alterra, Wageningen UR, Droevendaalsesteeg 3a, 6708 PB Wageningen, The Netherlands; 4. International Institute for Geo-Information Science and Earth Observation (ITC), P.O. Box 6, 7500 AA Enschede, The Netherlands

Submitted November 12, 2008; Accepted January 28, 2009; Electronically published May 7, 2009

ABSTRACT: The concentrations of resources in forage are not perfectly balanced to the needs of an animal, and food species differ in these concentrations. Under many circumstances, animals should thus forage on multiple food species to attain the maximum and most balanced intake of several resources. In this article we present a model to extend optimal foraging theory to incorporate concurrent foraging for multiple resources from several food species. A balancing of resources is achieved by representing the amount of a resource as the time during which it is used. Optimization is considered at two hierarchical levels: the time spent in a patch and the proportion of patches of each food species included in the foraging path. Our model results show that the balancing of resource intake can be achieved at the level of the foraging path, while the maximization of intake can be realized at the nested patch level. The choice for a food species should be dependent on the differences in intake and resource ratios between the food species. Under free choice of food species, the optimal patch residence time is subject not to differences between patches but to the local intake rate.

Keywords: optimal foraging theory, marginal value theorem, multiple nutrient requirements, diet composition, foraging scales.

\section{Introduction}

Optimal foraging theory (OFT) has focused mainly on the optimal acquisition of a single resource (generally energy as the principal resource for animals; Charnov 1976; Stephens and Krebs 1986; Newman et al. 1995; Bergman et al. 2001). Most extensions to OFT have involved the constraints animals face while trying to either maximize the intake rate of a resource or minimize the time needed to acquire a specific amount of this resource (Pulliam 1974, 1975; Westoby 1974; Belovsky 1978; Owen-Smith and Novellie 1983; Stephens and Krebs 1986; Illius and Gordon

* Corresponding author; e-mail: g.hengeveld@nioo.knaw.nl.

Am. Nat. 2009. Vol. 174, pp. 102-110. (C) 2009 by The University of Chicago. 0003-0147/2009/17401-50866\$15.00. All rights reserved.

DOI: $10.1086 / 598500$
1991; Owen-Smith 1993; Wilmshurst et al. 2000; Bergman et al. 2001; Fryxell et al. 2004). However, animals need to acquire more than one type of resource during foraging; many (macro)nutrients (e.g., proteins or nitrogen and phosphorus) are consumed and needed (Westoby 1974, 1978; Belovsky 1978; Prins and Beekman 1989; Prins 1996; Forbes 1999; Raubenheimer and Simpson 2004; Simpson et al. 2004; Anderson et al. 2005; Prins and van Langevelde 2008). The concentrations of these (macro)nutrients differ between and within the different types of food (Voeten and Prins 1999; Simpson et al. 2004; Klaassen and Nolet 2008; Prins and van Langevelde 2008). Although linear programming studies do take minimal requirements of several nutrients into account (Westoby 1974; Pulliam 1975; Belovsky 1978; Prins and Beekman 1989; Nolet et al. 1995; Voeten and Prins 1999), the optimal acquisition of multiple resources has rarely been considered quantitatively thus far (but see Simpson et al. 2004).

Herbivores have been shown to make foraging decisions at different scales. These decisions involve choosing in which areas to search for food, which food species to include in their foraging path or diet, and how long to stay in a given patch (Schoener 1971; Owen-Smith and Novellie 1983; Pyke 1984; Senft et al. 1987; Bailey et al. 1989; Skarpe et al. 2007). Most studies on optimal foraging focus on one of those decisions. In this article we extend OFT to include foraging for multiple resources at the scale of both the patch and the foraging path (i.e., a series of patches).

The turnover of (macro)nutrients (further referred to as resources) within an herbivore is continuous, with a relatively constant ratio between these resources (Forbes 1999; Klaassen and Nolet 2008; Prins and van Langevelde 2008). Yet, the acquisition of these resources is concentrated in short and discrete foraging bouts, while the concentration of resources varies from patch to patch. During these short foraging bouts, herbivores need to acquire enough of all resources to have the time to engage in 
Table 1: Model parameters

\begin{tabular}{|c|c|c|}
\hline Symbol & Units & Description \\
\hline$u_{i}$ & mass time ${ }^{-1}$ & Turnover rate of resource $i$ \\
\hline$c_{i j}$ & ... & Mass proportion of resource $i$ in food species $j$ \\
\hline$t_{j}$ & time & Time spent at a patch of food species $j$ \\
\hline$I_{j}\left(t_{j}\right)$ & mass & Mass intake from food species $j$ after spending $t_{j}$ time there \\
\hline $\bar{t}_{\mathrm{t}}$ & time & Average time taken to travel between two patches \\
\hline$D_{j}$ & & Proportion of the chosen patches of food species $j$ \\
\hline$g_{i}\left(t_{X}, t_{Y}\right)$ & mass time ${ }^{-1}$ & Global intake rate of resource $i$ \\
\hline$\gamma_{i}\left(t_{X}, t_{Y}\right)$ & ... & Ratio of intake rate to turnover rate of resource $i$ \\
\hline$\Gamma_{\min }\left(t_{X}, t_{Y}\right)$ & .. & Minimum of $\gamma_{i}\left(t_{X}, t_{Y}\right)$ for all resources $i$ \\
\hline$M_{j}$ & mass & Maximum intake at a patch of food species $j$ \\
\hline$h_{j}$ & time & Half-saturation constant of food species $j$ \\
\hline
\end{tabular}

nonforaging activities, such as vigilance and searching for mates. In food species, the ratio between the concentrations of resources will, in most cases, be different from the ratio of the turnover rates of these resources in the herbivore (Prins and Beekman 1989; Anderson et al. 2004; Prins and van Langevelde 2008). An herbivore thus faces the twofold problem of having to choose between different food species in order to balance the intake of several resources to its requirements and of having to maximize the rate at which this optimal food mix is consumed.

To address this problem, we use a stoichiometric approach within the optimal foraging paradigm (Sterner and Elser 2002; Simpson et al. 2004). Therefore, we link the composition of food species to the turnover rates of the different resources in the animal. The key element of our approach is that the consumed amount of each resource is expressed as the period during which it is used by the herbivore. This enables direct comparison between the intakes of the different resources and their use for the herbivore.

Time that is spent foraging cannot be spent on nonforaging activities; therefore, many optimal foraging models focus on either maximization of the intake rate or minimization of the foraging time (Stephens and Krebs 1986). When a consumer is foraging for several resources, the maximum intake rate of each resource separately is not necessarily met by the same decisions. Moreover, on timescales much longer than a few foraging bouts, for example, a couple of days, the consumption of a certain resource should be matched by the consumption of other resources. The time that then can be spent on nonforaging activities is determined by the resource for which the lowest intake rate relative to its turnover rate has been achieved. When maximizing the intake rate for the most limiting resource, the ratio between nonforaging time and foraging time is increased, ultimately leading to a maximum ratio between nonforaging time and foraging time. This would allow an animal to spend the maximum amount of time on (nonforaging) fitness-enhancing activities.

Imbalances in the diet, leading to excess in one or more resources, can be costly (Raubenheimer and Simpson 2004). For the initial analysis, these costs are neglected, to be included in the final part of the analysis. In this article we assume that the goal for the optimal forager is to maximize the time that can be spent on nonforaging activities, relative to the time spent foraging, that is, to minimize the long-term ratio of foraging time to nonforaging time. This minimization is reached by identifying the most limiting resource under given conditions and maximizing the rate of intake for this resource.

\section{Model}

Consider an herbivore and $p$ potential food species (denoted with subscript $j$ ). The food species contain $r$ resources (denoted with subscript $i$ ) that are used by the animal at a constant turnover rate $u_{i}$ (for a full list of the parameters and their descriptions, see table 1$)$. Let $c_{i j}$ denote the mass proportion of consumable resource $i$ in food species $j$. The food species are patchily distributed within the environment. All patches are considered to be of equal size and homogeneous, and each is considered to contain only one food species. The average travel time between two patches is $\bar{t}_{\mathrm{t}}$. The travel time is independent of the time spent in patches and of the ratio of patches in the foraging path (Charnov 1976). The cumulative consumption $\left(I_{j}\left(t_{j}\right)\right.$ at a patch of food species $j$ is strictly increasing with time in the patch $t_{j}$ and will typically be saturating asymptotically toward a maximum intake.

The herbivore can visit various patches of different food species while foraging. A proportion $D_{j}$ of all patches visited will be of food species $j$ (with $\sum_{j}^{p} D_{j}=1$ ). The global intake of resource $i$ is the weighted sum of the intake of that resource from each food species. The total time spent foraging is the weighted sum of the time spent foraging 
at patches of each food species plus the average travel time between the patches. The global intake rate of resource $i$ $\left(g_{i}\right.$, in units mass time $\left.{ }^{-1}\right)$ is the ratio of the global intake and the total time spent foraging:

$$
g_{i}=\frac{\sum_{j}^{p}\left(D_{j} c_{i j} I_{j}\left(t_{j}\right)\right)}{\bar{t}_{\mathrm{t}}+\sum_{j}^{p} D_{j} t_{j}} .
$$

Although every $g_{i}$ is expressed in the same units, these values cannot be compared in a straightforward way. Because the resources are used by the forager at different rates, a mass unit of one resource will have a totally different value for an animal than would the same mass of another resource. To sidestep this problem, we convert equation (1) into a ratio by dividing $c_{i j}$ by the turnover rate $u_{i}$. This gives

$$
\gamma_{i}=\frac{g_{i}}{u_{i}}=\frac{\sum_{j}^{p}\left[D_{j}\left(c_{i j} / u_{i}\right) I_{j}\left(t_{j}\right)\right]}{\bar{t}_{\mathrm{t}}+\sum_{j}^{p} D_{j} t_{j}} .
$$

Here $\left(\gamma_{i}-1\right)$ scales the time spent foraging to the time that the herbivore can continue without necessarily having to forage specifically for resource $i$. Conversely, $1 / \gamma_{i}$ is the fraction of the total time that the herbivore has to spend on foraging for resource $i$. Values of $\gamma_{i}$ below unity can thus be considered insufficient for survival because the consumption rate is less than the usage rate for this resource.

An optimal forager is assumed to maximize the intake rate for all resources. However, because there is no direct use for excesses of a resource, for two resources $A$ and $B$ increasing $\gamma_{A}$ beyond $\gamma_{B}$ will not increase the time for nonforaging activities. This means that time for nonforaging activities, represented by the scaling factor $\Gamma_{\min }$, is the minimum of all ratios $\gamma_{i}$ :

$$
\Gamma_{\min }=\min _{i}^{r}\left(\gamma_{i}\right)
$$

which is equivalent to the maximum fraction of the total time that has to be spent foraging $\left(1 / \Gamma_{\min }=\max _{i}^{r}\left(1 / \gamma_{i}\right)\right)$. To maximize the intake rate for all resources, the optimal forager should find the combination of $t$. and $D$. that maximizes $\Gamma_{\min }$ (and thus minimizes $1 / \Gamma_{\min }$ ), that is, to attain the maximum intake rate that is balanced to the turnover rates of the resources, given the available food species.

The function $\Gamma_{\min }$ contains two parameters that are under direct control of the herbivore: (1) $t_{j}$, the time spent in patches of food species $j$, and (2) $D_{j}$, the proportion of visited patches of food species $j$. The first control variable is a decision at the patch scale. The second control variable is the key determinant at the scale of the foraging path.

In the following we will evaluate the effect of simulta- neous optimization of foraging at these two scales, using the two control variables by applying numerical methods. For simplicity, but without loss of generality, we consider only two food species containing two resources. The presented conclusions are qualitatively similar to the results obtained for the case with more resources and food species. First, we will consider the optimal $t_{j}$ under a fixed $D_{j}$ (i.e., the herbivore cannot choose where to forage), and from there, we will continue to the case were the ratio of patch types is free to the herbivore and both $D_{j}$ and $t_{j}$ are optimized. We will discuss these results first with focus on the optimal value of $D_{j}$.

\section{Numerical Analyses}

Let us assume two food species ( $X$ and $Y$, both containing two resources, $A$ and $B$ ). An herbivore can forage on patches of both food species to attain the maximum and most balanced intake rate possible on both resources. To achieve this goal, the herbivore can decide how long to remain in a patch of any food species $\left(t_{X}\right.$ and $\left.t_{Y}\right)$, and it can alter the proportion of patches of each food species in the foraging path $\left(D_{X}\right.$ and $\left.D_{Y}=\left(1-D_{X}\right)\right)$. Using numerical methods, we will calculate the combination of $t_{X}$, $t_{Y}$, and $D_{X}$ that maximizes $\Gamma_{\text {min }}$.

Because the solution cannot be found analytically, an explicit form of the cumulative gain function $I_{j}\left(t_{j}\right)$ needs to be introduced. Without loss of generality, we use the Michaelis-Menten function because the optimal time in a patch predicted by the marginal value theorem can be derived explicitly for this function (see appendix). Then, $I_{j}\left(t_{j}\right)$ takes the form

$$
I_{j}\left(t_{j}\right)=\frac{M_{j} t_{j}}{h_{j}+t_{j}}
$$

with $M_{j}$ as the maximum intake at a patch of food species $j$ and $h_{j}$ as the half-saturation constant, that is, the time needed to acquire half of the maximum intake in the patch.

The numerical analyses of this model are carried out by first calculating the combination of $t_{X}$ and $t_{Y}$ that yields the highest $\Gamma_{\min }$ for all values of $D_{X} \in[0,1]$ (fig. 1). If the proportion of both resources is highest in one food species, $\Gamma_{\min }$ is maximized when the herbivore focuses only on this food species (fig. 1A, 1C). If both food species are complementary, by each species having a higher proportion of one resource relative to that of the other species, $\Gamma_{\min }$ is maximized for a mixture of both food species (fig. 1B).

The maximum $\Gamma_{\min }$ is determined by a combination of $t_{X}$ and $t_{Y}$. At the $D_{X}$ that yields the highest $\Gamma_{\min }, t_{j}$ for visited patches $\left(D_{j}>0\right)$ is equal to 



Figure 1: Optimal patch time at fixed $D_{X} . A-C$, Maximum $\gamma_{A}$ (dashed line), $\gamma_{B}$ (black line), and $\Gamma_{\min }$, the fraction of time for nonforaging activities (gray line), that can be reached at a given $D_{X}$ (numerical solution). $D-F, t_{X}$ and $t_{Y}$ corresponding to $\Gamma_{\min }$. The vertical dotted lines connect $D_{X}$ with maximum $\Gamma_{\min }$; the horizontal dotted line indicates the optimal value of $t_{j}$ as calculated with equation (5). Parameter values: $c_{A X}=0.075, c_{B X}=$ $0.025, u_{A}=u_{B}=1, M_{X}=M_{Y}=100, h_{X}=h_{Y}=1, \bar{t}_{\mathrm{t}}=2$. Left column, $c_{A Y}=0.03, c_{B Y}=0.01 ;$ middle column, $c_{A Y}=0.03, c_{B Y}=0.075 ;$ right column, $c_{A Y}=0.09, c_{B Y}=0.075$.

$$
t_{j}^{*}=\sqrt{h_{j} \bar{t}}
$$

(fig. $1 D-1 F$, dotted horizontal lines). This optimal solution is equal to the marginal value theorem prediction for foraging on a single resource in a single food species (Charnov 1976; see appendix). From equation (5) it follows that the ratio in optimal patch residence times for two food species is equal to the square root of the ratio of the half-saturation constants for those species:

$$
\frac{t_{X}^{*}}{t_{Y}^{*}}=\sqrt{\frac{h_{X}}{h_{Y}}} .
$$

For further analysis of the relationship between the proportion of the resources in the food species $\left(c_{\text {..) }}\right)$ and the optimal combination of food species $D_{X}$, we compare food species in pairs. These pairs consist of food species $X$ with given resource contents $c_{A X}$ and $c_{B X}$ and an alternative food species $Y$ from the whole range of alternative food species, with varying resource concentration $c_{\cdot Y} \in[0,1]$ and
$c_{A Y}+c_{B Y} \leq 1$. If we look at the values of $D_{X}$ and $t$., we find an interesting pattern (fig. 2). The combinations of specific values of both $D_{X}$ and $t$. can be categorized into five regions (fig. 2). These regions all represent different sets of alternative food species, relative to food species $X$. For each set of alternative food species, we will give an ecological interpretation (for the mathematical summary, see table 2).

Type I. The alternative food species $Y$ has lower proportions of both resources than does food species $X$. The optimal choice would be to choose only food species $X$. The optimal patch residence time is equal to the marginal value theorem prediction (Charnov 1976; appendix).

Type II. The alternative food species $Y$ has higher proportions of both resources. The optimal choice would be to always choose the alternative food species $Y$. The optimal patch residence time is equal to the marginal value theorem prediction.

Type III. Foraging on the alternative food species $Y$ will yield more of resource $A$ but less of resource $B$ than would foraging on food species $X$. However, food species $X$ pro- 


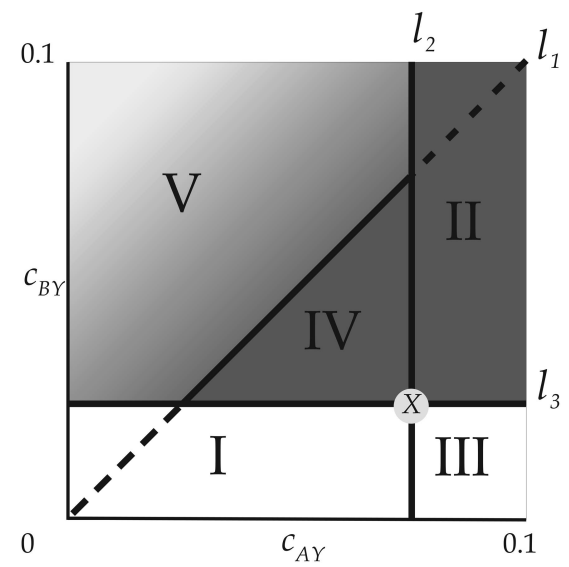

Figure 2: Schematic representation of the values of $D_{X}$, the optimal proportion of the diet that should consist of food species $X$ (on a scale from white for $D_{X}=1$ to dark gray for $D_{X}=0$ ). In the figure, one food species $X$ containing resources $A$ and $B$ in mass proportions $c_{A X}$ and $c_{B X}$ is compared to all alternative food species $Y$ for which $c_{. Y} \in[0,0.1]$. The $X$ marks the alternative food species $Y$, which has the same resource composition as food species $X$. The lines $l_{1}, l_{2}$, and $l_{3}$ (eqq. [7]-[9], respectively) delineate groups of alternative food species for which the optimal behavior in combination with food species $X$ is based on the same conditions (table 2). For two types of alternative food species (I, III) the optimal choice is for food species $X$; for two types (II, IV) the optimal choice is for food species $Y$; and for type $V$, there should be a mix of both food species, in a gradient that goes from small values of $D_{X}$ near the line $l_{1}$ to high values of $D_{X}$ farther away from this line. This gradient is described by equation (10). Parameter values: $c_{A X}=0.075$, $c_{B X}=0.025, u_{A}=u_{B}=0.1, M_{X}=M_{Y}=100, h_{X}=h_{Y}=1, \bar{t}_{\mathrm{t}}=2$.

vides more of resource $A$ than of resource $B$, making $B$ the most limiting resource. Therefore, including the alternative food species will not increase $\Gamma_{\text {min }}$. The optimal patch residence time is equal to the marginal value theorem prediction.

Type $I V$. Compared with food species $X$, food species $Y$ has less of resource $A$ and more of resource $B$. Because in both food species there is more of resource $A$ than of resource $B$, relative to the turnover rates of the herbivore, the optimal choice would be to leave food species $X$ for the alternative food species $Y$. The optimal patch residence time is equal to the marginal value theorem prediction.

Type $V$. The shortage of resource $B$ in food species $X$ can be compensated with a surplus of resource $B$ in food species $Y$, while the surplus of resource $A$ in food species $X$ can compensate for the shortage in food species $Y$. The optimal choice here is to feed on both food species. The optimal patch residence time for both food species is the product of the local half-saturation constant $\left(h_{j}\right)$ and the average travel time $\bar{t}_{\mathrm{t}}$ (see table 2 ).

These regions are separated by the lines $l_{1}, l_{2}$, and $l_{3}$, which are given, respectively, by the equations

$$
\begin{aligned}
& \frac{c_{A Y}}{c_{B Y}}=\frac{u_{A}}{u_{B}}, \\
& c_{A Y}=c_{A X} \frac{I_{X}\left(t_{X}^{*}\right)}{I_{Y}\left(t_{Y}^{*}\right)}, \\
& c_{B Y}=c_{B X} \frac{I_{X}\left(t_{X}^{*}\right)}{I_{Y}\left(t_{Y}^{*}\right)} .
\end{aligned}
$$

Line $l_{1}$ indicates those alternative food species $Y$ that contain a ratio of resources perfectly balanced to the ratio of the turnover rates of these resources (eq. [7]). Line $l_{2}$ indicates those alternative food species $Y$ for which the potential intake of resource $A$ is equal for both food species (eq. [8]). Line $l_{3}$ indicates those alternative food species $Y$ for which the potential intake of resource $i$ is equal for both food species (eq. [9]).

For the first four sets of alternative food species, only patches of one food species are visited. For the fifth set of alternative food species, the optimal diet consists of a mix of both food species. At the optimal combination of $X$ and $Y$, the condition $\Gamma_{\min }=\gamma_{A}=\gamma_{B}$ holds. In this region, the concentration of one resource is highest in one food species, while the concentration of the other resource is highest in the other food species $\left(c_{A Y}<c_{A X}\right.$ and $c_{B X}<c_{B Y}$; as in fig. 2). Therefore, the one $\gamma_{A}$ will be increasing with $D_{X}$, while the other will be decreasing with $D_{X}$ (in the example, $\gamma_{A}$ will be increasing). Because each food species has the highest concentration of one resource, $\Gamma_{\text {min }}$ will be at the intersection of the lines $\gamma_{A}$ and $\gamma_{B}$ with $D_{X}$ (fig. 1 ). Using equations (2) and (4), it follows that the optimal ratio of the proportion of patches of both food species can be calculated using the equation

$$
\frac{D_{X}}{D_{Y}}=\frac{I_{Y}\left(t_{Y}^{*}\right)\left[\left(c_{B Y} / u_{B}\right)-\left(c_{A Y} / u_{A}\right)\right]}{I_{X}\left(t_{X}^{*}\right)\left[\left(c_{A X} / u_{A}\right)-\left(c_{B X} / u_{B}\right)\right]} .
$$

Equation (10) is in agreement with Simpson et al. (2004), who show that patches of food species with resource contents that are balanced more to the herbivore's needs (in this case, $\left.\left(c_{B Y} / u_{B}\right)-\left(c_{A Y} / u_{A}\right)<\left(c_{A X} / u_{A}\right)-\left(c_{B X} / u_{B}\right)\right)$ are visited most frequently.

\section{Costs for Excess Intake}

Excess intake of resources, beyond the amount matched by the intake of the most limiting resource, can have negative effects on various aspects of the fitness of an animal (Raubenheimer and Simpson 2004; Anderson et al. 2005; Lee et al. 2008; Maklakov et al. 2008). Here we extend the model presented thus far by including costs for an excess intake of one of the resources.

Because excretion of the excess resource is costly, the 
Table 2: Types of alternative food species

\begin{tabular}{llccc}
\hline Type & \multicolumn{1}{c}{ Condition } & $D_{X}$ & $t_{X}$ & $t_{Y}$ \\
\hline I & $\left(c_{A Y}<c_{A X}\right) \wedge\left(c_{B Y}<c_{B X}\right)$ & 1 & $\sqrt{h_{X} \bar{t}_{\mathrm{t}}}$ & $\ldots$ \\
II & $\left(c_{A Y}>c_{A X}\right) \wedge\left(c_{B Y}>c_{B X}\right)$ & 0 & $\ldots$ & $\sqrt{h_{Y} \bar{t}_{\mathrm{t}}}$ \\
III & $\frac{c_{A Y}}{u_{A}}>\frac{c_{A X}}{u_{A}}>\frac{c_{B X}}{u_{B}}>\frac{c_{B Y}}{u_{B}}$ & 1 & $\sqrt{h_{X} \bar{t}_{\mathrm{t}}}$ & $\ldots$ \\
IV & $\frac{c_{A X}}{u_{A}}>\frac{c_{A Y}}{u_{A}}>\frac{c_{B Y}}{u_{B}}>\frac{c_{B X}}{u_{B}}$ & 0 & $\ldots$ & $\sqrt{h_{Y} \bar{t}_{\mathrm{t}}}$ \\
$\mathrm{V}$ & $\left(c_{A Y}<c_{A X}\right) \wedge\left(c_{B Y}>c_{B X}\right) \wedge\left(\frac{c_{A Y}}{u_{A}}<\frac{c_{B Y}}{u_{B}}\right) \wedge\left(\frac{c_{A X}}{u_{A}}>\frac{c_{B X}}{u_{B}}\right)$ & Eq. (10) & $\sqrt{h_{X} \bar{t}_{\mathrm{t}}}$ & $\sqrt{h_{Y} \bar{t}_{\mathrm{t}}}$ \\
\hline
\end{tabular}

total gain (in our case $\Gamma_{\min }$ ) should be diminished as a function of the imbalance of the diet. To approximate this effect, we rewrite equation (3) as

$$
\Gamma_{\min , c}=\min _{i}^{r}\left(\gamma_{i}\right)-\sum_{k}^{p} \alpha_{k}\left[\gamma_{k}-\min _{i}^{r}\left(\gamma_{i}\right)^{2}\right]
$$

where $\alpha_{i}$ weights the cost of having resource $i$ in excess. The excess $\left(\gamma_{k}-\min _{i}^{r}\left(\gamma_{i}\right)\right)$ is squared to represent increased costs for larger excess (Anderson et al. 2005).

Let us return to the case with two resources $(A$ and $B)$ and two food species $\left(X\right.$ and $Y$ ) and set $\alpha_{A}=\alpha_{B}=\alpha$. When $\alpha$ increases, a new subdivision of regions I and II occurs: the one patch type is more balanced to the turnover rates but less lucrative than the other (fig. 3). In region III, mixing $X$ with $Y$ cannot decrease the imbalance of consuming only $X$ because $X$ is already more balanced to the turnover rates than is $Y$. In region IV, mixing $Y$ with $X$ cannot decrease the imbalance of consuming only $Y$ because $Y$ is already more balanced to the turnover rates than is $X$. In region $V$, mixing is such that there is no excess of any resource $\gamma_{A}=\gamma_{B}$.

The subdivision of regions I and II is along the lines $l_{1}$ (eq. [7]), $l_{4}$, which shifts from $l_{3}$ (for $\alpha \sim 0$ ) to $l_{4 \text {,ext }}$ for very high values of $\alpha$, and $l_{5}$, which shifts from $l_{1}$ (for $\alpha \sim 0$ ) to $l_{3}$ for very high values of $\alpha$. Thus, $l_{4, \text { ext }}$ is given by

$$
\frac{c_{A Y}}{c_{B Y}}=\frac{c_{A X}}{c_{B X}}
$$

These lines divide the regions into three subregions (fig. $3)$. For these subregions the changes in the optimal $D_{X}$ that occur when $\alpha$ is increased are different. Regions Ia and IIa do not change with increasing $\alpha$. Regions $\mathrm{Ib}$ and IIb change gradually with increasing $\alpha$ : region Ib from an optimum at $D_{X}=1$ to an optimum of $D_{X}=0$ at high values of $\alpha$ and region IIb from an optimum at $D_{X}=0$ to an optimum at $D_{X}=1$. Regions Ic and IIc change to an optimum of $0<D_{X}<1$. For all regions the optimal time spent foraging in a patch is equal to equation (5).

\section{Discussion}

In this article we present a model that predicts the foraging behavior that maximizes and balances intake when foraging for multiple resources on several food species. It predicts that when animals are free to choose the food species they feed from, patch residence time is determined only by local information, namely, the local intake rate and the average travel time to reach a patch. Furthermore, the ratio of food species in the foraging path is dependent only on food species characteristics, namely, the concentration of resources and the intake rate that can be attained on this food species.

This model enables the prediction of the optimal combination of food species in a method that is complementary to the work of explicitly defining a multidimensional fitness landscape (Raubenheimer and Simpson 2004; Simpson et al. 2004) or considering independent fitness effects of surpluses or deficits in separate resources (Anderson et al. 2004; Raubenheimer and Simpson 2004; Simpson et al. 2004). Our model can be tested using field experiments. Furthermore, the model predicts the patch residence time in patches of several food species when multiple resources are taken into account. Although the model is presented here for only two resources and two food species, the results shown here hold for more resources and more food species.

The model presented in this article extends the commonly used single-resource approach in OFT (Charnov 1976; Stephens and Krebs 1986; Owen-Smith 1993; Fryxell et al. 2004). This single-resource approach is applicable whenever there is one resource limiting for all combinations of food species (fig. 2, sets of alternative food species I, II, III, and IV). In these situations, one food species is chosen exclusively. There is, however, a large range of alternative food species (fig. 2, region V) for which none of the resources is limiting in both food species (Belovsky 1978; Prins and Beekman 1989; Voeten and Prins 1999; Raubenheimer and Simpson 2004; Simpson et al. 2004; Ludwig et al. 2007). Here the ratio between the resources 



Figure 3: Influence of costs for excess intake of resources on $D_{X}$. Both panels are built up analogous to figure 2. Region I is subdivided into subregions Ia, where no changes occur; Ib, which changes from $D_{X}=1$ to $D_{X}=0$ in one part of the region and to $0<D_{X}<1$ in the rest of the region; and Ic, which changes from $D_{X}=1$ to $0<D_{X}<1$. Region II is subdivided into subregions IIa, where no changes occur; IIb, which changes to $0<D_{X}<1$; and IIc, which changes to $D_{X}=1$ for one part of the region and $0<D_{X}<1$ for the rest. These subregions are delineated by the lines $l_{1}$ (eq. [7]), $l_{4}$, and $l_{5} ; l_{4}$ (right) is equal to $l_{4, \text { ext }}$ (eq. [12]). Left, results for a low value of $\alpha(\alpha=0.005)$; right, results for a high value of $\alpha(\alpha=$ 0.1). Parameter values: $c_{A X}=0.075, c_{B X}=0.025, u_{A}=u_{B}=0.1, M_{X}=M_{Y}=100, h_{X}=h_{Y}=1, \bar{t}_{\mathrm{t}}=2$.

in the diet can be optimized by selecting patches of both food species. We provide a model to investigate the optimal foraging behavior for these combinations of food species at two foraging scales simultaneously. Our explicit solution for the case of two food species can easily be expanded to include multiple food species.

At the scale of the foraging path, the forager optimizes the balance in its intake by controlling the proportion of the patches of each food species within its path. The balance between the proportions of food species $X$ and food species $Y$ in the foraging path is determined by the ratio of the intake at patches of both food species and the differences of the resource contents within each food species relative to the turnover rates (eq. [10]).

So far, predicting herbivore food selection from the resource content of forage plants has proven to be difficult (Owen-Smith and Novellie 1983; Skarpe et al. 2007). The model presented in this article provides new testable hypotheses about food species selection in the foraging path.

Within a patch, the optimal patch residence time depends on the average travel time between patches and the shape of the cumulative intake within the patch over time. This information can be considered to be locally available. Within an optimal foraging path, the predicted optimal patch residence time is the same as the marginal value theorem prediction for one food species (see appendix; Charnov 1976). We show that this is the optimal patch residence time even when more than one food species is included in the forage path, as long as the forager can select at both the path level of foraging and the patch level of foraging. From the specific shape of the cumulative intake function, we predict that the ratio of patch residence times is determined by the ratio between the halfsaturation constants of these patches (eq. [6]). The shape of the cumulative intake over time in patches of different food species (i.e., the half-saturation constant) is readily assessed from field data (Illius et al. 1999; Skarpe et al. 2007).

An imbalanced diet (i.e., when the intake of one resource exceeds the intake of other resources relative to the rate at which they are used) can be costly to animals (Raubenheimer and Simpson 2004; Anderson et al. 2005; Lee et al. 2008; Maklakov et al. 2008). Costs associated with excess resource intake could thus influence the choice for alternative food species. Yet, our analysis shows that excess costs influence only a very limited set of the potential food species. As in the situation without costs of excess resource intake, balancing of intake (and in this situation the minimization of excess resource intake) will take place through the selection of patches, while the optimal time spent foraging in a patch is accurately represented by the marginal value theorem (eq. [5]; appendix).

Studies of optimal foraging behavior aim to understand the ultimate goals of foraging animals (Stephens and Krebs 1986). The proximate mechanisms through which these goals can be reached remain untouched in this article. Such mechanisms could be flexible, for example, depending on the nutritional state of the animal, or they could be behavioral syndromes tuned to a predictable environment. So far, we have considered nonchanging environments. In 
changing environments, however, the potential food species available to an animal can change, resulting in a change in the optimal diet for this animal. Moreover, we have explored only the effect of short-term costs of excess in intake. As shown, even with these costs for excess resource intake, the range of potential food species for which the diet can be optimized is large. Most gradual changes in the forage species composition of the environment will thus be within the range where an optimal diet can be composed. In this range it is the proximate mechanism of diet choice that limits the possibilities of an animal to reach this optimal diet, for example, satiation when a certain resource is consumed. Outside this range, excesses in the uptake of one resource cannot be compensated for by balancing food species. An unbalanced diet can also have long-term fitness consequences (Lee et al. 2008; Maklakov et al. 2008). Such fitness consequences could make environments in which balancing is impossible unfavorable for survival or reproduction, in which case environments that can provide food species for sufficient balancing need to be included in the animal's home range.

Most parameters in our model can be measured directly in experimental studies (Illius et al. 1999; Skarpe et al. 2007). Most difficult will be the assessment of the turnover rates for the different resources $\left(u_{i}\right)$. Turnover rates of water, energy, and nitrogen are quite well known, but those of other elements such as Se, Co, P, K, and $\mathrm{Na}$ are much less readily available, except for some domestic herbivores (Prins and van Langevelde 2008).

We have shown that the modeling framework presented in this article allows for extensions to meet specific criteria. In this article we discuss incorporation of costs of imbalances in the diet. Other extensions could be the possibility of conversion of one nutrient for another, as is known for protein that can yield energy (Raubenheimer and Simpson 2004), or the effect of polyphenolics that reduce the efficiency of intake of nutrients (Jansman 1993; Van Soest 1994).

We presented an optimal foraging approach to the challenge of achieving a balanced diet and a maximum intake for multiple resources by selecting on two scales. Balancing of resources is achieved by representing the amount of a resource as the time within which it is used by the animal. We identify the rules that make pairs of food species exclusive or complementary. For complementary food species, the optimal balance is achieved by taking into account the difference in intake of the resources in patches of both food species. Both when visiting patches of multiple food species and when visiting one single food species, patch residence time is dependent only on the shape of the local cumulative intake function and the average traveling time between patches.

\section{Acknowledgments}

We would like to thank A. Brodin, F. de Boer, J. Fryxell, I. Heitkonig, J. McNamara, B. Nolet, H. Prins, S. Simpson, and S. van Wieren for comments on an earlier version of this manuscript. This is publication 4472 for the Netherlands Institute of Ecology (NIOO-KNAW).

\section{APPENDIX}

\section{The Marginal Value Theorem with One Food Species}

Considering one food species $(X)$ containing two resources ( $A$ and $B), D_{X}=1$. We write equation (2) as

$$
\gamma_{i}=\frac{\left(c_{i X} / u_{i}\right) I_{X}\left(t_{X}\right)}{t_{X}+\bar{t}_{t}}
$$

If $\left(c_{A X} / u_{A}\right)>\left(c_{B X} / u_{B}\right)$

$$
\Gamma_{\min }=\gamma_{B}=\frac{\left(c_{B X} / u_{B}\right) I_{X}\left(t_{X}\right)}{t_{X}+\bar{t}_{\mathrm{t}}} .
$$

The marginal value theorem (Charnov 1976) predicts that any patch should be left whenever

$$
\frac{\partial\left(c_{B X} / u_{B}\right) I_{j}\left(t_{j}\right)}{\partial t_{j}}=\Gamma_{\min }^{*}
$$

where $\Gamma_{\min }^{*}$ is the minimum $\gamma_{i}$ when $t_{X}$ is optimal. Using equation (4), we can write

$$
\frac{\partial\left(c_{B X} / u_{B}\right) I_{X}\left(t_{X}\right)}{\partial t_{X}}=\frac{\left(c_{B X} / u_{B}\right) M_{X} h_{X}}{\left(h_{X}+t_{X}\right)^{2}} .
$$

Combining equations (A2)-(A4), we get

$$
\frac{c_{B X}}{u_{B}} \frac{\left[M_{X} t_{X}^{*} /\left(h_{X}+t_{X}^{*}\right)\right]}{\left(t_{X}^{*}+\bar{t}_{\mathrm{t}}\right)}=\frac{c_{B X}}{u_{B}} \frac{M_{X} h_{X}}{\left(h_{X}+t_{X}^{*}\right)^{2}},
$$

which we can reduce to

$$
t_{X}^{*}=\sqrt{h_{X} \bar{t}_{\mathrm{t}}}
$$

\section{Literature Cited}

Anderson, T. R., M. Boersma, and D. Raubenheimer. 2004. Stoichiometry: linking elements to biochemicals. Ecology 85:1193-1201. Anderson, T. R., D. O. Hessen, J. J. Elser, and J. Urabe. 2005. Metabolic stoichiometry and the fate of excess carbon and nutrients in consumers. American Naturalist 165:1-15.

Bailey, D. W., L. R. Rittenhouse, R. H. Hart, D. M. Swift, and R. W. Richards. 1989. Association of relative food availabilities and locations by cattle. Journal of Range Management 42:480-482. 
Belovsky, G. E. 1978. Diet optimization in a generalist herbivore: the moose. Theoretical Population Biology 14:105-134.

Bergman, C. M., J. M. Fryxell, C. C. Gates, and D. Fortin. 2001. Ungulate foraging strategies: energy maximizing or time minimizing? Journal of Animal Ecology 70:289-300.

Charnov, E. L. 1976. Optimal foraging, the marginal value theorem. Theoretical Population Biology 9:129-136.

Forbes, J. M. 1999. Natural feeding behaviour and feed selection. Pages 3-12 in D. van der Heide, E. A. Huisman, E. Kanis, J. W. M. Osse, and M. W. A. Verstegen, eds. Regulation of feed intake. CABI, Wallingford.

Fryxell, J. M., J. F. Wilmshurst, and A. R. E. Sinclair. 2004. Predictive models of movement by Serengeti grazers. Ecology 85:2429-2435.

Illius, A. W., and I. J. Gordon. 1991. Prediction of intake and digestion in ruminants by a model of rumen kinetics integrating animal size and plant characteristics. Journal of Agricultural Science 116:145157.

Illius, A. W., I. J. Gordon, D. A. Elston, and J. D. Milne. 1999. Diet selection in goats: a test of intake rate maximization. Ecology 80: 1008-1018.

Jansman, A. J. M. 1993. Tannins in feedstuffs for simple-stomached animals. Nutrition Research Reviews 6:209-236.

Klaassen, M., and B. A. Nolet. 2008. Stoichiometry of endothermy: shifting the quest from nitrogen to carbon. Ecology Letters 11: 785-792.

Lee, K. P., S. J. Simpson, F. J. Clissold, R. Brooks, J. W. O. Ballard, P. W. Taylor, N. Soran, and D. Raubenheimer. 2008. Lifespan and reproduction in Drosophila: new insights from nutritional geometry. Proceedings of the National Academy of Sciences of the USA 105:2498-2503.

Ludwig, F., H. de Kroon, and H. Prins. 2007. Impacts of savanna trees on forage quality for a large African herbivore. Oecologia (Berlin) 155:487-496.

Maklakov, A. A., S. J. Simpson, F. Zajitschek, M. D. Hall, J. Dessmann, F. Clissold, D. Raubenheimer, R. Bonduriansky, and R. C. Brooks. 2008. Sex-specific fitness effects of nutrient intake on reproduction and lifespan. Current Biology 18:1062-1066.

Newman, J. A., A. J. Parsons, J. H. M. Thornley, P. D. Penning, and J. R. Krebs. 1995. Optimal diet selection by a generalist grazing herbivore. Functional Ecology 9:255-268.

Nolet, B. A., P. J. Van der Veer, E. G. J. Evers, and M. M. Ottenheim. 1995. A linear programming model of diet choice of free-living beavers. Netherlands Journal of Zoology 45:315-337.

Owen-Smith, N. 1993. Assessing the constraints for optimal diet models. Evolutionary Ecology 7:530-531.

Owen-Smith, N., and P. Novellie. 1983. What should a clever ungulate eat? American Naturalist 19:151-178.

Prins, H. H. T. 1996. Ecology and behaviour of the African buffalo. Chapman \& Hall, London.
Prins, H. H. T., and J. H. Beekman. 1989. A balanced diet as a goal of grazing: the food of the Manyara buffalo. African Journal of Ecology 27:241-259.

Prins, H. H. T., and F. van Langevelde. 2008. Assembling a diet from different places. Pages 129-155 in H. H. T. Prins and F. van Langevelde, eds. Resource ecology: spatial and temporal dynamics of foraging. Springer, Dordrecht.

Pulliam, H. R. 1974. On the theory of optimal diets. American Naturalist 108:59-74.

1975. Diet optimization with nutrient constraints. American Naturalist 109:765-768.

Pyke, G. H. 1984. Optimal foraging theory: a critical review. Annual Review of Ecology and Systematics 15:523-575.

Raubenheimer, D., and S. J. Simpson. 2004. Organismal stoichiometry: quantifying non-independence among food components. Ecology 85:1203-1216.

Schoener, T. W. 1971. Theory of feeding strategies. Annual Review of Ecology and Systematics 2:369-404.

Senft, R. L., M. B. Coughenour, D. W. Bailey, L. R. Rittenhouse, O. E. Sala, and D. M. Swift. 1987. Large herbivore foraging and ecological hierarchies. BioScience 37:789-798.

Simpson, S. J., R. M. Sibly, K. P. Lee, S. T. Behmer, and D. Raubenheimer. 2004. Optimal foraging when regulating intake of multiple nutrients. Animal Behaviour 68:1299-1311.

Skarpe, C., I. Jansson, L. Seljeli, R. Bergstrom, and E. Roskaft. 2007. Browsing by goats on three spatial scales in a semi-arid savanna. Journal of Arid Environments 68:480-491.

Stephens, D. W., and J. R. Krebs. 1986. Foraging theory. Princeton University Press, Princeton, NJ.

Sterner, R. W., and J. J. Elser. 2002. Ecological stoichiometry: the biology of elements from molecules to the biosphere. Princeton University Press, Princeton, NJ.

Van Soest, P. J. 1994. Nutritional ecology of the ruminant. 2nd ed. Cornell University Press, Ithaca, NY.

Voeten, M. M., and H. H. T. Prins. 1999. Migratory ungulates: is the grass really greener at the other side? Pages 61-82 in M. M. Voeten, ed. Living with wildlife: coexistence of wildlife and livestock in an east African savanna system. $\mathrm{PhD}$ thesis. Wageningen University.

Westoby, M. 1974. An analysis of diet selection by large generalist herbivores. American Naturalist 108:290-304.

- 1978. What are the biological bases of varied diets? American Naturalist 112:627-631.

Wilmshurst, J. F., J. M. Fryxell, and C. M. Bergman. 2000. The allometry of patch selection in ruminants. Proceedings of the Royal Society B: Biological Sciences 267:345-349.

Associate Editor: Axel G. Rossberg Editor: Donald L. DeAngelis 